EPJ Web of Conferences 71, 00109 (2014)

DOI: 10.1051/epjconf/20147100109

(C) Owned by the authors, published by EDP Sciences, 2014

\title{
Subthreshold $\phi$ meson production in nucleus-nucleus collisions
}

\author{
K. Piasecki for the FOPI Collaboration ${ }^{1, a}$ \\ ${ }^{1}$ Institute of Experimental Physics, Faculty of Physics, University of Warsaw, Poland
}

\begin{abstract}
Part of emission of the negatively charged kaons from the nucleus-nucleus collisions appears to originate from decays of $\phi(1020)$ mesons into the $\mathrm{K}^{+} \mathrm{K}^{-}$channel. We report on the measurement of the emission yield of $\phi$ mesons in the collisions of $\mathrm{Ni}+\mathrm{Ni}$ at the beam kinetic energy of $1.91 \mathrm{~A} \mathrm{GeV}$. The experiment was done with the FOPI spectrometer at the SIS 18 accelerator at GSI, Darmstadt. The total yield of $\phi$ was found to be $\left(4.5 \pm 0.7_{-1.2}^{+1.8}\right) \cdot 10^{-4}$ per triggered event. Ratio of $\phi / \mathrm{K}^{-}$emission yields was estimated at $0.42 \pm 0.13_{-0.11}^{+0.17}$, meaning that about $20 \%$ of negatively charged kaons emitted from the collision originate from decays of $\phi$ mesons. The results are in agreement with similar HADES results done for the collisions of $\mathrm{Ar}+\mathrm{KCl}$.
\end{abstract}

\section{Introduction}

Nucleus-nucleus collisions offer a unique possibility of studying the properties of particles in hot and dense hadronic environment. At beam energies of about $1-2 \mathrm{~A} \mathrm{GeV}$, accessible at the SIS 18 synchrotron in GSI, Darmstadt, the hadronic degrees of freedom are dominant, and the channel of strangeness production opens up. A joint experimental and theoretical effort has shown that basic properties of kaons (mass, lifetime, and dispersion relation) may change to some extent as a result of interaction with nuclear matter [1]. To verify this, patterns of kinematical observables like directed and elliptic flow, and $\mathrm{K}^{-} / \mathrm{K}^{+}$ratio have been compared to the predictions of various transport models of nucleus-nucleus collisions, where the kaon-nucleon interaction was expressed either in terms of the potential or the G-matrix formalism [2]. Positively (negatively) charged kaons were found to undergo slight repulsion from (attraction to) centers of nuclear matter. However, it has already been suggested that the sample of $\mathrm{K}^{-}$mesons might have a considerable admixture from the decays of $\phi$ mesons $[3,4]$.

$\phi(1020)$ meson is composed of strange quarks (s-sbar). About half of decays of this particle proceed via the $\mathrm{K}^{+} \mathrm{K}^{-}$channel $(B R=48.9 \%$ [5]). As the mean decay path of this particle in vacuum, $c \tau \approx 46 \mathrm{fm}$ [5], is larger than the size of the nucleus, the decays occur predominantly in vacuum. This is additionally supported by the fact that due to the short mean free path of $\mathrm{K}^{-}$[6], the decays of $\phi$ occuring inside the collision zone are to a large extent non-reconstructable from their products.

The total emission yield of positively charged kaons is about 30-50 times larger than that of $\mathrm{K}^{-}$ [7] at the beam energies below $2 \mathrm{~A} \mathrm{GeV}$, so the decays of $\phi$ contribute more significantly to $\mathrm{K}^{-}$than to $\mathrm{K}^{+}$. Since negatively charged kaons originating from $\phi$ decays are predominantly created in vacuum, in principle they should not be treated together with $\mathrm{K}^{-}$emitted directly from hot and dense fireball.

ae-mail: krzysztof.piasecki@fuw.edu.pl 


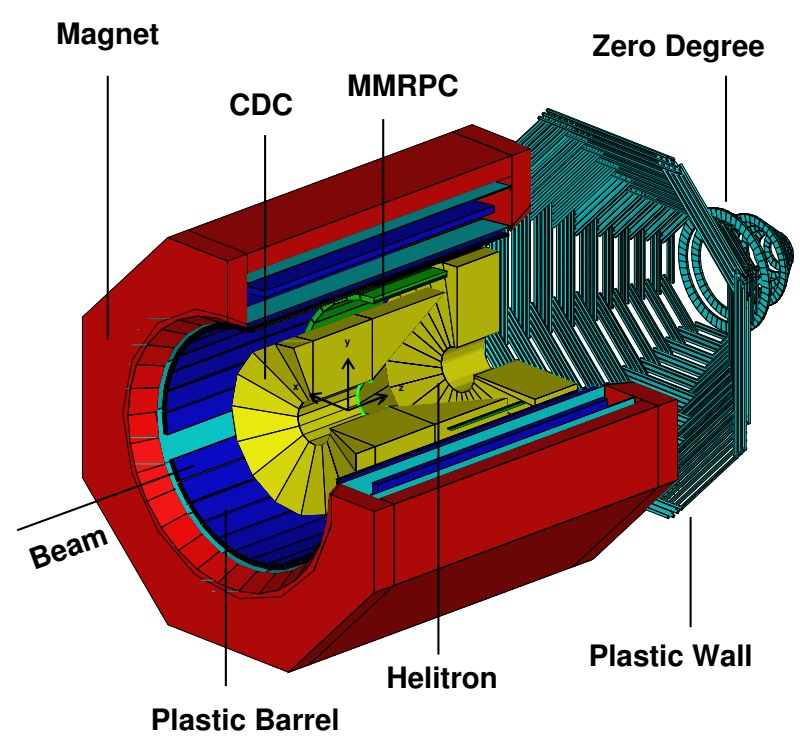

Figure 1. FOPI experimental setup

The threshold energy for the production of $\phi$ in a free nucleon-nucleon collision is $2.6 \mathrm{GeV}$. Thus, for the abovementioned region of beam energies, the production channels are the sub-threshold ones. Transport calculations performed in the framework of the BUU model for the collisions of $\mathrm{Ni}+\mathrm{Ni}$ at the beam kinetic energy of $1.93 \mathrm{~A} \mathrm{GeV}$ hint about the importance of channels involving the excited baryonic states ( $\mathrm{BB} \rightarrow \mathrm{BB} \phi, \mathrm{B}=\{\mathrm{N}, \Delta\}$ ) and mesons: $\rho \mathrm{B} \rightarrow \mathrm{B} \phi, \pi \mathrm{B} \rightarrow \mathrm{B} \phi$, and $\rho \pi \rightarrow \phi$, while the contribution from the $\mathrm{K}^{+} \mathrm{K}^{-} \rightarrow \phi$ channel seems to be negligible. Also, a considerable absorption of $\phi$ mesons is predicted via the $\phi N \rightarrow K \Lambda$ process [8,9].

The experimental data on the emission yields and kinematical patterns of $\phi$ is very scarce. At the beam kinetic energies below $10.8 \mathrm{~A} \mathrm{GeV} \mathrm{[10],} \mathrm{and} \mathrm{in} \mathrm{particular} \mathrm{in} \mathrm{the} \mathrm{subthreshold} \mathrm{region,} \mathrm{the}$ first sample of $\phi$ mesons produced below the threshold was found by the FOPI Collaboration in the central collisions of $\mathrm{Ni}+\mathrm{Ni}$ at $1.93 \mathrm{~A} \mathrm{GeV}$. However, only 23 events were obtained above background [3]. Two more measurements were reported: $168 \phi$ mesons detected in $\mathrm{Ar}+\mathrm{KCl}$ collisions at 1.756A $\mathrm{GeV}$ [4], and, recently, $108 \phi$ 's from $\mathrm{Al}+\mathrm{Al}$ at $1.9 \mathrm{~A} \mathrm{GeV}$ [11]. In this work, preliminary results of the $\phi$-meson measurement in $\mathrm{Ni}+\mathrm{Ni}$ collisions at $1.91 \mathrm{~A} \mathrm{GeV}$ are presented.

\section{FOPI spectrometer}

The FOPI spectrometer has been installed at the beamline of the SIS 18 synchrotron at GSI (Darmstadt). It is designed for fixed target experiments at beam energies within $0.1 \mathrm{~A}-2 \mathrm{~A} \mathrm{GeV}$. The complete detector set-up covers nearly the full solid angle. The innermost detectors are two drift chambers: Central Drift Chamber (CDC) and Helitron, serving as tracking devices. The CDC covers a wide range of polar angles, $23^{\circ}<\theta_{\text {lab }}<113^{\circ}$, counted with respect to the target used for this experiment. 
The CDC is surrounded by two detectors in the barrel geometry, dedicated for the Time-of-Flight (ToF) measurement, the Plastic Scintillation Barrel (PSB), covering $55^{\circ}<\theta_{l a b}<110^{\circ}$, and the Multistrip Multi-gap Resistive Plate Chamber (RPC), spanning $30^{\circ}<\theta_{l a b}<53^{\circ}$. The latter detector is characterized by the high granularity and timing resolution [13], superior to the performance of the PSB. The abovementioned detectors are operated in the $0.6 \mathrm{~T}$ magnetic field generated by the superconducting solenoid magnet. At the front of FOPI the Plastic scintillation Wall (PlaWa) is installed. This detector helps to constrain the collision centrality by measuring the multiplicity of emitted charged products. The more detailed description of FOPI apparatus can be found in [12].

\subsection{Particle identification}

The measurement of momentum (p), charge (q) and velocity (v) of an emitted particle is performed using a combined information from the CDC and one of detectors measuring the Time-of-Flight (PSB or RPC). Charged particles are identified by extracting the mass from the momentum and velocity relation.

Neutral particles characterized by decay paths smaller than the outer radius of the CDC can be reconstructed within decay channels where all the products are charged. E.g. FOPI allows for the reconstruction of $\phi$ meson in the dominant $\mathrm{K}^{+} \mathrm{K}^{-}$channel. While the clear identification of $\mathrm{K}^{+}\left(\mathrm{K}^{-}\right)$ within the PSB detector is limited to the momenta of $0.6(0.38) \mathrm{GeV}$, the significantly better granulation and timing resolution of the RPC device results in enhancing the momentum limit up to over 1.2 $\mathrm{GeV} / \mathrm{c}\left(\mathrm{K}^{+}\right)$and $0.8 \mathrm{GeV} / \mathrm{c}\left(\mathrm{K}^{-}\right)$, thus revealing most of their dynamic range (see Fig. 13 in ref. [13]).

\section{The experiment}

${ }_{28}^{58} \mathrm{Ni}$ ions of intensity $8 \times 10^{5}$ ions/s were accelerated in the SIS 18 synchrotron to the kinetic energy of $1.91 \mathrm{~A} \mathrm{GeV}$. The beam irradiated the $360 \mathrm{mg} / \mathrm{cm}^{2}$-thick ${ }_{28}^{58} \mathrm{Ni}$ target with interaction probability of about $1 \%$. The trigger based on multiplicity of activated modules in the PlaWa (PMUL $\geq 5$ ) and PSB (BMUL $\geq 1$ ) selected the sample of central events amounting to about $50 \%$ of the total geometrical cross section. Assuming the sharp cut-off dependency between the cross section and maximum impact parameter $\left(b_{\max }\right)$, and a simple geometrical model of interpenetrating spheres, the number of participants averaged over the impact parameter was estimated to be $\left\langle A_{\text {part }}\right\rangle_{b}=53$. The number of accepted events was about $7.6 \times 10^{7}$.

\subsection{Reconstruction of $\phi$ mesons}

The kinematical variables of candidates of charged kaons and $\phi$ mesons were limited to the ranges denoted in Table 1 . The cuts applied to $\phi$ meson candidates roughly correspond to the geometrical acceptance of the FOPI setup.

The invariant mass distribution of the $\mathrm{K}^{+} \mathrm{K}^{-}$pairs reveals a strong maximum around $1.0195 \mathrm{GeV} / \mathrm{c}^{2}$, the nominal mass of the $\phi$ meson (cf. Fig. 2). The combinatorial background was obtained using the mixed events technique. After subtracting the background, $173 \pm 14 \phi$ mesons were found with a significance level of 8.7 .

In order to evaluate the acceptance and efficiency correction, the GEANT [14] detector simulation package was used. $\phi$ mesons were generated according to the Siemens-Rasmussen formula [15], describing the phase space distribution of a particle emitted from a source of temperature $\mathrm{T}$, and radially expanding with velocity $\beta \mathrm{c}$. The T parameter was varied between 70 and $130 \mathrm{MeV}$, and $\beta \mathrm{c}$ between 0 and $0.3 \mathrm{c}$. Differences in the efficiency correction due to initial parameters were further included in the 
Table 1. Kinematical cuts applied to charged kaon and $\phi$ meson candidates.

\begin{tabular}{ll}
\hline & $\mathrm{K}^{ \pm}$ \\
\hline$\vartheta_{\text {Lab }}$ & $\in\left(28^{\circ}, 90^{\circ}\right)$ \\
$\mathrm{p}_{\mathrm{K}}^{\mathrm{RPC}}$ & $<1.20 \mathrm{GeV} / \mathrm{c}$ \\
$\mathrm{p}_{\mathrm{K}}^{\mathrm{PS}}$ & $<0.72 \mathrm{GeV} / \mathrm{c}$ \\
$\mathrm{p}_{\mathrm{K}-}^{\mathrm{RPC}}$ & $<0.85 \mathrm{GeV} / \mathrm{c}$ \\
$\mathrm{p}_{\mathrm{K}-}^{\mathrm{PSB}}$ & $<0.60 \mathrm{GeV} / \mathrm{c}$ \\
\hline \multicolumn{2}{c}{$\phi$} \\
\hline$\vartheta_{C M}^{N N}$ & $\in\left(95^{\circ}, 150^{\circ}\right)$ \\
$T_{C M}^{N N}$ & $\in(0.15,0.75) \mathrm{GeV}$ \\
\hline
\end{tabular}

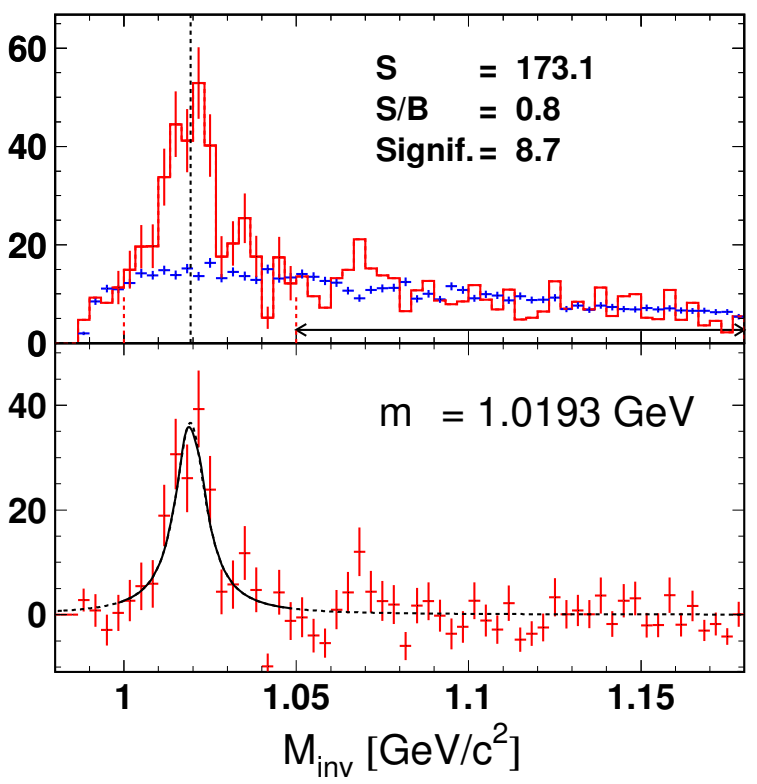

Figure 2. Upper panel: Invariant mass distribution of $\mathrm{K}^{+} \mathrm{K}^{-}$pairs (red) and the reconstructed combinatorial background (blue), normalized in the region denoted by the arrow. Lower panel: invariant mass distribution after subtraction of background.

systematic uncertainties of the investigated physics variables. $\phi$ mesons were subsequently embedded in the events generated by the IQMD model [16], simulating the collisions of $\mathrm{Ni}+\mathrm{Ni}$ at the beam kinetic energy of $1.91 \mathrm{~A} \mathrm{GeV}$.

\subsection{Results}

The total yield of emitted $\phi$ mesons was found to be $\left(4.5 \pm 0.7\right.$ (stat.) ${ }_{-1.2}^{+1.8}$ (syst.) $) \cdot 10^{-4}$ per triggered event. An estimation of the $\phi / \mathrm{K}^{-}$ratio of yields can be made, taking into account the total $\mathrm{K}^{-}$emission yield of $(2.1 \pm 0.4) \cdot 10^{-3}$ measured by KaoS [17] at the same beam energy, but for the centrality selection corresponding to $0<b<4.4 \mathrm{fm}$. Assuming the experimentally obtained scaling dependency 
with the mean number of participants as $\mathrm{A}_{\text {part }}^{1.8 \pm 0.3}$ [18], the $\mathrm{K}^{-}$yield at centrality selection for the $\phi$ measurement presented in this work would be $(1.1 \pm 0.3) \cdot 10^{-3}$.

The $\phi / \mathrm{K}^{-}$ratio can be thus preliminarily estimated to $0.42 \pm 0.13_{-0.11}^{+0.17}$. The result is similar to the value of $0.37 \pm 0.13$ found for the collisions of $\mathrm{Ar}+\mathrm{KCl}$ at the beam kinetic energy of $1.756 \mathrm{~A} \mathrm{GeV}$ [4]. Taking into account the branching ratio of the $\phi \rightarrow \mathrm{K}^{+} \mathrm{K}^{-}$, our estimation means that about $20 \%$ of emitted $\mathrm{K}^{-}$are the products of $\phi$ meson decays.

\section{Summary}

FOPI has studied the emission of $\phi(1020)$ mesons from the collisions of $\mathrm{Ni}+\mathrm{Ni}$ at the beam kinetic energy of $1.91 \mathrm{~A} \mathrm{GeV}$ in the region of phase space corresponding to $95^{\circ}<\vartheta_{C M}^{N N}<150^{\circ}$.

The trigger collisions from most central ones up to $50 \%$ of the total cross section. The $\phi$ emission yield extrapolated to the full solid angle was found to be $\left(4.5 \pm 0.7\right.$ (stat.) ${ }_{-1.2}^{+1.8}$ (syst.) $) \cdot 10^{-4}$ per triggered event.

The ratio of $\phi$ to $\mathrm{K}^{-}$yields was estimated to be $42 \pm 13_{-0.11}^{+0.17} \%$. This translates into the fraction of about $20 \%$ of negatively charged kaons originating from the decays of $\phi$ mesons. As the latter ones are predominantly produced in vacuum, this result should be included in the studies of the modification of properties of $\mathrm{K}^{-}$in hot and dense hadronic medium. The obtained results are also consistent with the HADES data from the collisions of $\mathrm{Ar}+\mathrm{KCl}$ at the beam kinetic energy of $1.756 \mathrm{~A} \mathrm{GeV}$.

\section{References}

[1] C. Fuchs et al., Prog. Part. Nucl. Phys. 56, 1 (2006)

[2] C. Hartnack, H. Oeschler, Y. Leifels, E.L. Bratkovskaya, J. Aichelin, Phys. Rep. 510, 119 (2012)

[3] A. Mangiarotti et al., Nucl. Phys. A 714, 89 (2003)

[4] C. Agakishiev et al., Phys. Rev. C 80, 025209 (2009)

[5] J. Beringer et al. (Particle Data Group), Phys. Rev. D 86, 010001 (2012)

[6] C.B. Dover, G.E. Walker, Phys. Rep. 89, 1 (1982)

[7] A. Foerster et al., Phys. Rev. C 75, 024906 (2007)

[8] H.W. Barz, Zétényi, Gy. Wolf, B. Kämpfer, Nucl. Phys. A 705, 223 (2002)

[9] B. Kämpfer, R. Kotte, C. Hartnack, J. Aichelin, J. Phys. G: Nucl. Part. Phys. 28, 235 (2002)

[10] B.B. Back et al., Phys. Rev. C 69, 054901 (2004)

[11] P. Gasik, Ph. D. Thesis, University of Warsaw, 2011; P. Gasik, K. Wiśniewski, T. Matulewicz, Acta Phys. Pol. B 41, 379 (2010)

[12] J. Ritman et al., Nucl. Phys. B, Proc. Suppl. 44, 708 (1995) B. Sikora, Acta Phys. Pol. B 31 (2000) 135

[13] M. Kiš et al., Nucl. Instr. and Meth. A 646, 27 (2011)

[14] http://wwwasdoc.web.cern.ch/wwwasdoc/geant_html3/geantall.html

[15] P.J. Siemens, J.O. Rasmussen, Phys. Rev. Lett. 42, 880 (1979)

[16] C. Hartnack et al., Eur. Phys. J. A 1, 151 (1998)

[17] M. Menzel et al., Phys. Lett. B 495, 26 (2000)

[18] R. Barth et al., Phys. Rev. Lett. 78, 4007 (1997) 